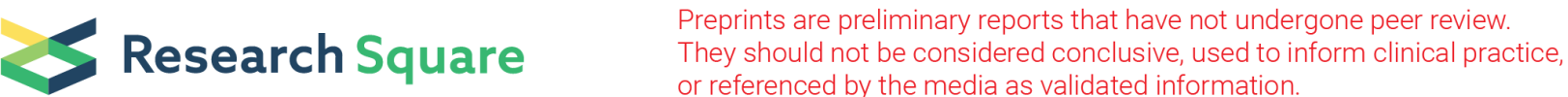

\section{Interferon regulatory factor 8 induces alveolar macrophage hypoxia/reoxygenation injury via the PINK1/Parkin mitophagy pathway}

\section{Lin Zhang}

Wuhan University Renmin Hospital

\section{Bo Hao}

Wuhan University Renmin Hospital

Ruyuan He

Wuhan University Renmin Hospital

Zilong Lu

Wuhan University Renmin Hospital

Haojie Feng

Wuhan University Renmin Hospital

Xiaokang Shen

Wuhan University Renmin Hospital

Ziyao Zhang

Wuhan University Renmin Hospital

Wenyang Jiang

Wuhan University Renmin Hospital

Wei Wang

Wuhan University Renmin Hospital

Tao Fan

Wuhan University Renmin Hospital

Qing Geng ( $\square$ gengqingwhu@whu.edu.cn )

Wuhan University Renmin Hospital

\section{Research}

Keywords: macrophage, hypoxia/reoxygenation, IRF8, lung ischemia-reperfusion injury, autophagy

Posted Date: July 16th, 2020

DOI: https://doi.org/10.21203/rs.3.rs-43520/v1 
License: (c) (i) This work is licensed under a Creative Commons Attribution 4.0 International License. Read Full License 


\section{Abstract}

Background: Lung ischemia-reperfusion injury (LIRI) is a complex process and macrophages play a central role in it. Interferon regulatory factor 8 (IRF8) is a transcription factor in the innate immune system, which, together with autophagy, are important components of the mechanisms of LIRI. We aimed to explore the regulatory role of IRF8 in hypoxia/reoxygenation (HR) injury and PINK1-mediated mitophagy.

Methods: Cultured NR8383 macrophages were subjected to HR as an in vitro model of LIRI. Cell viability, cell damage and inflammation during HR were evaluated by Cell Counting Kit-8 assay, lactate dehydrogenase assessment and enzyme linked immunosorbent assay, respectively. Meanwhile, PINK1/Parkin-mediated mitochondrial autophagy was detected by quantitative real-time PCR, western blot, transmission electron microscopy and double immunofluorescence labeling method. The effects of IRF8 and PINK1 on cell injury, inflammatory response and autophagy during HR were assessed using IRF8 short-hairpin RNA plasmids and PINK1 overexpression plasmids, respectively.

Results: IRF8 inhibition was able to alleviate cell injury, inflammatory response and autophagy while PINK1 overexpression could exacerbate them. Moreover, IRF8 downregulation could reduce the expression of PINK1 in HR injury and attenuate the effects of it.

Conclusion: IRF8 was able to induce HR injury via the PINK1/Parkin mitophagy pathway. Inhibition of IRF8 and PINK1/Parkin-mediated mitophagy might be a potential target for the treatment of LIRI.

\section{Background}

Ischaemia and reperfusion of lung is a commonly occurring process in a variety of medical situations, including lung transplantation, sleeve lobectomy, pulmonary thromboendarterectomy, and aortic surgery[1]. Among them, lung transplantation remains to be the only treatment for end-stage pulmonary disease patients. However, lung ischemia-reperfusion injury (LIRI) is a key event that contributes to primary graft dysfunction (PGD), a major cause of morbidity and mortality after lung transplantation, and remains to be the leading cause of death after lung transplantation[2]. Thus, understanding of the mechanisms of this disease is extremely important for the improvement of the prognosis of lung transplantation. The mechanisms of LIRI is complex and involves many aspects such as innate immune response, autophagy, oxidative stress and endoplasmic reticulum stress[3].

Interferon regulatory factors (IRFs) are transcription factors that induces and regulates expressions of interferon and interferon-related signaling pathways. IRFs can be expressed downstream of the signaling pathways in the innate immune and the adaptive immune system; they could also participate in driving inflammatory responses and regulating the differentiation of immune cells[4]. Human IRFs are comprised of 9 family members, namely IRF1-9. With their central role in type I Interferon (IFN- $a$ and - $\beta$ ) biology, IRFs have been implicated in the pathophysiology of several inflammatory and autoimmune conditions, such as systemic lupus erythematosus[5]. However, the participation of IRFs in LIRI has not been studied in 
any previous research yet. Interferon regulatory factor 8 (IRF8), a member of the IRF family, has been shown to be a regulator of autophagy, a process involved in LIRI[6]. Meanwhile, IRF8 could also regulate the induction of type I interferon genes downstream of pattern recognition receptors, whose activation is thought to be an important part of the mechanism of ischemia-reperfusion lung injury[3]. Therefore, IRF8 is likely to have participated in this process.

Autophagy is an evolutionary highly conversed catabolic process among all eukaryotes ranging from yeast to mammals, providing nutrients for vital cellular functions during fasting and other forms of stress[7]. Autophagy was mostly characterized to be activated through the inhibition of mammalian target of rapamycin (mTOR) and has been elucidated to be either a nonselective process or a selective process that eliminate harmful materials or excess cell organs such as damaged mitochondria[7]. It is known that mitochondrial autophagy (mitophagy) is often caused by exposure to hypoxia and participates in multiple aspects of tissue homeostasis, exerting an essentially physiological effects on eukaryotic cells[8]. More recently, mitophagy has been proved to participate in the process of LIRI by regulating the mTOR pathway[9]. However, regulation of hypoxia/reoxygenation (HR) injury by phosphatase and tensin homologue (PTEN)-induced putative kinase 1 (PINK1), one of the most studied pathway molecule in mitophagy with Parkin functioning downstream of it, has not been substantiated yet. Therefore, the present study aims to elucidate the role of IRF8 in HR injury and its regulation on the PINK1/Parkin mitophagy pathway in that situation.

\section{Methods}

\subsection{Cell culture and establishment of an HR model}

The current study used a rat alveolar macrophage cell line NR8383 (ATCC, CRL-2192) as an in vitro model in consideration of the important role alveolar macrophage activation plays in the development of LIRI[10]. The cells were maintained in F-12K medium (Thermo Fisher, Waltham, MA, USA) at $37^{\circ} \mathrm{C}$ in a humidifed $5 \% \mathrm{CO} 2$ atmosphere. In addition, the medium contained $10 \%$ fetal calf serum. When the cells reached $80 \%$ confluence, they were digested with $0.25 \%$ trypsin. An HR model of NR8383 cells was established for mimicking lung ischemia-reperfusion injury as described previously[11]. Briefly, the cells were cultured in a humidified incubator with $0.5 \%$ oxygen for $6 \mathrm{~h}$ for hypoxia treatment, The cells were subsequently moved to a normoxic incubator and cultured for another $6 \mathrm{~h}$ as the reoxygenation treatment. The cells normally cultured for the same time length without HR treatment were treated as a normal control (NC) group.

\subsection{Knock-down of IRF8 and overexpression of PINK1 by transfection}

To down-regulate IRF8 expression, cells were transfected with IRF8 short-hairpin RNA (shRNA) plasmids (sh-IRF8, BioFavor, Wuhan, China) with Lipofectamine 2000 (Invitrogen, Carlsbad, CA, USA) before HR treatment. Meanwhile, negative control plasmids (sh-NC, BioFavor, Wuhan, China) were applied to transfect cells. In addition, transfection of PINK1 overexpression plasmids (OE-PINK1, BioFavor, Wuhan, China) was carried out using Lipofectamine 2000 (Invitrogen, Carlsbad, CA, USA) to up-regulate the 
expression of PINK1 in macrophages before HR treatment. Cells were harvested $48 \mathrm{~h}$ after transfection and used for further analysis. The cells normally cultured for the same time length without transfection before HR treatment were treated as an HR group.

\subsection{Immunofluorescence}

For the identification of macrophages, immunofluorescence was applied. Cells were grown on glass coverslips and fixed with $4 \%$ paraformaldehyde for $15 \mathrm{~min}$, washed by PBS thrice, permeabilized in $0.5 \%$ Triton X-100 (Beyotime, Shanghai, China) for 20 min, washed by PBS thrice and blocked by goat serum (Boster, Wuhan, China) for $30 \mathrm{~min}$. The cells were then incubated with primary antibodies (anti-CD68, 1:100, Abcam, Cambridge, UK) overnight at $4^{\circ} \mathrm{C}$ followed by incubation with Cyt3-labeled secondary antibodies (Boster, Wuhan, China) for $1 \mathrm{~h}$ in the dark at $37^{\circ} \mathrm{C}$. DAPI (Beyotime, Shanghai, China) was then applied to stain the cell nuclei for $5 \mathrm{~min}$ in the dark. The stained cells were observed and photographed under an Olympus microscope (BX53, Olympus, Tokyo, Japan). Identification of macrophages by immunofluorescence was shown in Figure $1 \mathrm{~A}$ with cells observed to expressed CD68, a surface marker of macrophages.

\subsection{Cell Counting Kit-8 (CCK8) assay}

Cell proliferation was determined using the CCK8 (Beyotime, Shanghai, China) assay according to the

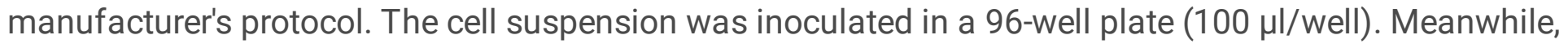
blank wells were filled with equal amount of PBS. The plate was cultured overnight in a humidified incubator with $5 \% \mathrm{CO} 2$ in air at $37^{\circ} \mathrm{C}$. Next, the CCK-8 solution was added $(10 \mu \mathrm{l} /$ well), and the plate was cultured at $37^{\circ} \mathrm{C}$ for $4 \mathrm{~h}$. Finally, the Multiskan MK3 microplate reader (Multiskan MK3, Thermo Fisher, Waltham, MA, USA) was employed to measure the optical density values at the wavelength of $450 \mathrm{~nm}$ (OD450).

\subsection{Lactate dehydrogenase (LDH) assessment}

LDH release, an index that reflects cell damage, was assessed by LDH assay kit (Nanjing Jiancheng Bioengineering Institute, Nanjing, China), according to manufacturer's protocol. Briefly, the cell supernatant was mixed with supplied assay buffer and absorbance readings were obtained at the wavelength of $450 \mathrm{~nm}$ (OD450) using the Multiskan MK3 microplate reader (Multiskan MK3, Thermo Fisher, Waltham, MA, USA). The LDH activity was presented as fold changes compared to control absorbance.

\subsection{Enzyme linked immunosorbent assay (ELISA)}

The cytokine levels in the supernatant were measured using commercial ELISA kits (TNF- $\alpha$ and IL-1 $\beta$ ELISA kits, USCN, Wuhan, China; IL-6 and IL-10 ELISA kits, Elabsicence, Wuhan, China) following the instructions.

\subsection{Quantitative real-time PCR (qPCR)}


Total RNA from NR8383 cells were prepared by using TRIzol (Thermo Fisher, Waltham, MA, USA) according to the manufacturer's instructions and then reversely transcribed to cDNA with the Hiscript Reverse Transcriptase (Vazyme, Nanjing, China). The resulting cDNAs were quantified by qPCR with Taq Plus DNA Polymerase (Tiangen, Beijing, China) according to the manufacturer's instructions. Real-time PCR was performed using SYBR Green Master Mix (Vazyme, Nanjing, China) on the QuantStudio 6 RTPCR system (QuantStudio 6, ABI, Waltham, MA, USA), and mRNA expressions were normalized to GADPH expressions and fold changes were calculated by the $2^{-\Delta \Delta C q}$ method. Sequences of primers were as follows:

\begin{tabular}{llll}
\hline Name & Forward(5'-3') & Reverse (5'-3') & Size \\
\hline Rat GAPDH & ACAGCAACAGGGTGGTGGAC & TTTGAGGGTGCAGCGAACTT & $253 \mathrm{bp}$ \\
Rat PINK1 & GACCGCTACCGCTTCTTCCG & TCTCCTCGATCAGCCCCAAC & $148 \mathrm{bp}$ \\
Rat PRKN & GGCTGTGGGTTCGTTTTCTG & GGCTTGGTGGTTTCTTGAT & $176 \mathrm{bp}$ \\
Rat IRF8 & GTCACGGAGATGGAGTGTGG & CTGGGATAAGGCTGAATGGT & $216 \mathrm{bp}$ \\
\hline
\end{tabular}

\subsection{Western blot}

Cell lysates were subjected to SDS-PAGE and transferred to polyvinylidene fluoride (PVDF) membranes. The membranes were blocked with $5 \%$ skim milk in TBST for $2 \mathrm{~h}$ and then incubated with diluted (1:1000) primary antibodies against LC3 (Affinity Biosciences, OH, USA), PINK1 (ABclonal, Wuhan, China), Parkin (Abcam, Cambridge, UK), IRF8 (Thermo Fisher, Waltham, MA, USA) and GAPDH (Goodhere, Hangzhou, China) overnight, washed with TBST, followed by being stained with anti-rabbit or anti-mouse IgG (Boster, Wuhan, China). Immunoreactivity was visualized by enhanced chemiluminescence kit (ECL kit, Applygen, Beijing, China). Band quantification was performed by densitometry analyses using Image $\mathrm{J}$ software (National Institutes of Health, USA).

\subsection{Transmission electron microscopy}

Autophagy was marked by the emergence of autophagy flux, represented by emerging autophagosomes evidenced by transmission electron microscopy (TEM). Thus, TEM was applied for observation of autophagosomes. Briefly, the cell mass were fixed with $2.5 \%$ glutaraldehyde in $0.1 \mathrm{M}$ phosphate buffer at $4^{\circ} \mathrm{C}$ for $2 \mathrm{~h}$. After fixation and dehydration, ultrathin sections $(60-80 \mathrm{~nm})$ were obtained. All the sections were stained with lead citrate and uranyl acetate followed by detection under an FEl microscope (FEI Tecnai G20, FEl, Hillsboro, OR, USA).

\subsection{Double immunofluorescence labeling}

Mitophagy initiation was determined based on the increased co-localization of mitochondria and PINK1 proteins. Therefore, detection of mitophagy was performed by double immunofluorescence labeling 
method. Cells were incubated with MitoTracker ${ }^{\circledR}$ Deep Red FM (Yeasen, Shanghai, China) at $37^{\circ} \mathrm{C}$ for 30 min for mitochondrial labeling. Cells were then grown on glass coverslips and embedded in paraffin. Specimens were incubated overnight at $4{ }^{\circ} \mathrm{C}$ and stained for PINK1 (anti-PINK1, ABclonal, Wuhan, China) immunofluorescence. An FITC-labeled secondary antibody (Boster, Wuhan, China) was employed as the secondary antibody for PINK1. The cell nuclei were then stained with DAPI (Beyotime, Shanghai, China) for $5 \mathrm{~min}$ in the dark. Sections were examined and photographed using an Olympus microscope (BX53, Olympus, Tokyo, Japan).

\subsection{Statistical analyses}

Statistical analyses were performed with the GraghPad Prism 7 software (GraphPad, San Diego, CA, USA). Measurement data were expressed as means \pm SD. One-way analysis of variance (ANOVA) was applied for analyses of differences among multiple groups. Differences with P 0.05 were considered statistically significant.

\section{Results}

\subsection{Successful downregulation of IRF8 and overexpression of PINK1 expressions}

In order to investigate the role of IRF8 and PINK1 in HR injury, We generated IRF8 knock-down macrophages using sh-IRF8 and PINK1 overexpression macrophages using OE-PINK1. IRF8 knock-down and PINK1 overexpression in the cells were confirmed by western blot analysis (Figure 1B-D). Interestingly, the inhibition of IRF8 expression could suppress the expression of PINK1 protein while the overexpression of PINK1 had no significant effect on IRF8 protein expression (Figure 1B-D), suggesting a regulatory role of IRF8 on PINK1 proteins.

\subsection{IRF8 inhibition alleviates cell injury and the inflammatory response during HR}

HR injury has been considered to cause inhibited cell viability and cell death [11]. Thus, in order to investigate the role of IRF8 in HR-mediated cell injury, CCK8 assay and LDH test were performed. HR treatment significantly inhibited cell viability and aggravated cell damage (Figure 2A,B). Noticeably, significantly higher cell viability was observed in sh-IRF8-treated cells undergoing HR treatment compared with sh-NC controls as assessed by CCK8 assay (Figure 2A). Consistent with these findings, IRF8 downregulation also significantly reduced the elevated level of LDH in macrophages after HR treatment (Figure 2B). Compared with HR group, PINK1-over-expressing macrophages exhibited further inhibited cell viability and exacerbated cell damage compared with those in the HR group (Figure 2A,B). Furthermore, the effects of PINK1 overexpression on cell viability and cell damage were weakened by sh-IRF8 cotransfection (Figure 2A, B). Together, these findings show that IRF8 downregulation alleviates HRmediated cell insult that could be exacerbated by PINK1 overexpression.

During lung ischemia-reperfusion injury, the inflammatory response is initiated during the ischemic phase and is an essential factor in the pathophysiological process. Therefore, we aimed to investigate whether 
IRF8 could affect the inflammatory response during HR injury in macrophages. ELISA analyses indicated that the levels of proinflammatory cytokines, namely TNF-a, IL-1 $\beta$, IL-6, were significantly elevated in HR group compared to those in the NC group cells (Figure 2C-E). Moreover, the expression levels of antiinflammatory cytokine IL-10 in the supernatant was significantly lower in the HR group compared with that in the NC group (Figure 2F). To further examine whether IRF8 directly influences inflammation in macrophages, we detected the expressions of the above cytokines in cells transfected with sh-NC or shIRF8. As evidenced by the results, macrophages with sh-IRF8 transfection exhibited lower levels of the proinflammatory cytokines and higher levels of the antiinflammatory cytokine than the sh-NC controls after HR challenge (Figure 2C-F). Moreover, higher expressions of TNF-a, IL-1 $\beta$ and IL- 6 were also evidenced in OE-PINK1 group cells compared with those in HR group (Figure 2C-E). Consistently, PINK1 upregulation weakened the expression of IL-10 in HR-induced inflammation and the effects of PINK1 overexpression on inflammation were also weakened by sh-IRF8 co-transfection (Figure 2C-F).

\subsection{IRF8 inhibition reduces autophagy in response to HR}

Lung ischemia-reperfusion injury affects the expressions of autophagy-related proteins and promotes autophagy $[9,12]$. Thus, we examined whether IRF8 directly regulates autophagy and facilitates its damaging effects on HR injury. Compared with the NC group cells, the level of autophagy was elevated in the HR group cells, as demonstrated by the increased autophagosomes observed by transmission electron microscope (Figure 3A). Moreover, the ratio of autophagy related proteins LC3-II/I, an index indicating the level of autophagy, was significantly increased in HR group cells compared with NC group cells (Figure 3B, C). IRF8 downregulation could decrease the level of autophagy in macrophages, as evidenced by the decreased autophagosomes observed by transmission electron microscope and the reduced level of LC3-II/I in sh-IRF8 group cells compared to HR group cells (Figure 3A-C). The results indicated a regulatory effect of IRF8 on autophagy induced by HR treatment. Furthermore, autophagosome formation was increased in OE-PINK1 group compared with HR group and sh-IRF8 cotransfection in RE-PINK1 group partially decreased it compared to cells transfected with OE-PINK1 after HR (Figure 3A). Consistently, sh-IRF8 co-transfection decreased the level of LC3-II/I that had been upregulated by OE-PINK1 after HR (Figure 3B, C), implicating a regulatory role of IRF8 on PINK1-mediated autophagy during HR.

\subsection{IRF8 deficiency alleviates mitochondrial autophagy during HR}

PINK1/Parkin-mediated mitochondrial autophagy is a classical mitochondrial autophagy pathway and has also been proven to be involved in ischemia-reperfusion injury and HR injury[9, 13-15]. Therefore, to evidence the involvement of PINK1-mediated mitophagy, immunofluorescence PINK1/MitoTracker double labeling was employed to assess mitophagy in macrophages. MitoTracker is a fluorescent probe indicating the distribution of mitochondria. Compared with NC group, HR group presented with increased colocalized fluorescence intensity of mitochondrial autophagy protein PINK1 and MitoTracker; while in the sh-IRF8 group, the colocalization was reduced compared to HR group (Figure 4). Moreover, sh-IRF8 co- 
transfection decreased the colocalization of PINK1/mitochondria increased by OE-PINK1 transfection (Figure 4).

\subsection{IRF8 deficiency attenuates PINK1/Parkin mitophagy pathway induced by HR}

To further confirm the participation of PINK1/Parkin mitophagy pathway in HR-induced cell injury, western blot and qPCR analyses were performed. As evidenced by westernblot analyses, the levels of PINK1 and Parkin were significantly increased by HR treatment, which were downregulated by sh-IRF8 transfection. Co-transfection of sh-IRF8 in RE-PINK1 group partially decreased PINK1 and Parkin expressions that had been upregulated by OE-PINK1 after HR despite that the difference of Parkin expressions between OE-PINK1 group and RE-PINK1 group was not significant (Figure 5A-C). Interestingly, the expression of IRF8 protein that was increased by HR treatment was decreased by sh-IRF8, as it was under normoxia conditions (Figure 1B,C), but it had not been significantly influenced by OE-PINK1 (Figure $5 A, D)$. Consistent with the above results, the mRNA levels of PINK1 and PRKN were also significantly increased after HR treatment and were downregulated by sh-IRF8 transfection; co-transfection of sh-IRF8 in RE-PINK1 group partially decreased PINK1 and PRKN expressions that had been upregulated by OEPINK1 after HR (Figure 5E,F). Also, the expression of IRF8 mRNA that was increased by HR treatment was decreased by sh-IRF8 and was not significantly influenced by OE-PINK1 (Figure 5G). The above results indicated that IRF8 functions upstream of the PINK1/parkin mitophagy pathway during HR-induced cell injury.

\section{Discussion}

Although plenty of studies have employed different techniques to study the pathophysiology associated with lung ischemia-reperfusion injury, the specific pathophysiological mechanisms of it remain incompletely understood. Studies from our laboratory have demonstrated the role of autophagy, endoplasmic reticulum stress and ubiquitin-proteasome system in HR injury or LIRI[11, 16, 17]. Other mechanisms include oxidative stress, innate immune responses and so on[3]. However, the regulatory role of IRF8 in HR injury or LIRI has not been explored yet. The current study demonstrated the regulatory role of IRF8 on cell viability, cell damage and inflammatory response through the PINK/Parkin mitophagy pathway during $\mathrm{HR}$.

Lung ischemia-reperfusion injury is associated with multiple diseases and needs to be addressed in situations such as lung transplantation[18]. The understanding of LIRI is critical to the improvement of the prognoses of lung transplantation. However, data correlated to the molecular and cellular mechanisms underlying hypoxic and reperfusion lung damage are limited. Moreover, the majority of the studies are focused on endothelial and epithelial cells rather than alveolar macrophages that have critical immunological functions in acute lung injuries including ischemia reperfusion lung injury[10, 17, 19, 20]. In the present study, we utilized an in vitro HR model of macrophages, as applied in previous research, to mimic LIRI[21]. Indeed, when sh-IRF8 was applied to inhibit IRF8 expression, alveolar macrophages showed increased cell viability and alleviated cell damage compared with sh-NC after HR. OE-PINK1, 
which was used to upregulate PINK1 expressions, had a reversed effect on cell injury. These observations indicated a scathing role of IRF8 and PINK1 in HR-induced injury of alveolar macrophages.

IRF8 is one of the members of the IRF family, which possess an N-terminal DNA binding domain (DBD) for the recognition of a special DNA sequence, and a C-terminal IRF-associated domain (IAD) for proteinto-protein interactions[4]. Especially, IRF8 is an important transcription factor that acts downstream of innate and adaptive immune signaling pathways, it plays a critical role in the differentiation, polarization, and activation of mononuclear phagocytic cells. IRF8 has been characterized, in previous research, to be a central element and functions downstream of the IFN- $y$ signaling pathway that involves IFN- $y$ 's binding to the receptor STAT1 and is recognized to regulate the activation of the proinflammatory M1 phenotype macrophages[22]. Consistently, IRF8 activation was also proved to induce M1 polarization of macrophages after advanced glycation endproducts treatment previously[23]. Moreover, IRF8 could be controlled by the Notch-RBP-J signaling pathway to participate in the regulation of TLR-induced inflammatory polarization of macrophages, namely the M1 macrophages polarization[24]. In the current study, the results revealed that IRF8 inhibition alleviated inflammation in macrophages, as evidenced by decreased expression of proinflammatory cytokines and increased expression of antiinflammatory cytokines after sh-IRF8 transfection, which was consistent with the previous research. Our study was also in consistence with previous findings demonstrating that IRF8 was the target of histone deacetylase SIRT1 to regulate LPS-induced inflammation in macrophages and that IRF8 could promote inflammatory response in macrophages during encephalomyelitis $[25,26]$. In addition, IRF8 was corroborated to be a regulator of autophagy in previous studies; it could activate autophagy in cutaneous wound healing and promote autophagy in macrophages to clear invading bacteria[6,23]. Our study was accordant with previous ones, as reflected by the decreased autophagy levels in cells transfected with sh-IRF8 undergoing HR treatment.

Autophagy is a highly conserved catabolic process induced under various conditions of cellular stress. It could prevent cell damage, promote cell survival in the shortage of energy or nutrient and respond to various cytotoxic insults[7]. However, the effects of autophagy on ischemia-reperfusion injury or HR injury vary in different situations. Liu et al. revealed that the inhibition of autophagy could protect lung from ischemia-reperfusion injury[27]. Ye et al. also demonstrated that enhanced autophagy was associated with aggravated HR injury in mouse pulmonary microvascular endothelial cells[28]. In contrast, our previous research proved that autophagy could decrease apoptosis in rat alveolar macrophages exposed to HR and decrease apoptosis and inflammation in rat LIRI[16, 17]. In the present study, enhanced autophagy was associated with exacerbated inflammation and cell injury in macrophages. These different conclusions showing opposite effects of autophagy in similar disease models may lead to a confusion about the effects of autophagy on cytotoxicity. The contradictory results might be explained by the different degrees of autophagy in these studies. Mild autophagy could exert a protective effect on cells while excessive autophagy may lead to cell death[29, 30].

As a form of selective autophagy, mitochondrial autophagy could be induced through various pathways. Among them, PINK1/Parkin is a classical one. It has been elucidated that PINK1 accumulates at the outer 
membrane of mitochondria (OMM) and recruits Parkin when mitochondria are damaged. Accumulated PINK1 phosphorylates ubiquitin on Ser65, with Ub-pSer65 binding to Parkin, leading to its 'open conformation' that can be phosphorylated by PINK1 on its Ser65 and being fully activated. Ubiquitinated OMM proteins can then recruit mitophagy receptors containing an LC3 interacting region (LIR) motif to interact with the LC3 anchored on the membrane of autophagosomes and lead to mitochondrial autophagy[31]. Consistently, the effects of PINK1-mediated mitophagy on LIRI also varied. The upregulation of PINK1 and the scathing role of mitophagy in LIRI were corroborated in previous research[9]. However, some research also illustrated that improved inflammatory response and apoptosis in cardiomyocyte HR injury was achieved through activation of the PINK1/Parkin mediated mitophagy[13]. In the current study, the upregulation of PINK1 expression promoted autophagy, cell injury and inflammation in HR-treated cells, which could be partially alleviated by IRF8 knock-down. The inconsistency among the studies might also be explained by the different degrees of mitochondrial autophagy in them.

Overall, our study indicated that HR induces PINK1/Parkin mediated mitophagy, which was associated with increased inflammation and exacerbated cell injury and was regulated by IRF8 in macrophages; by inhibiting the expression of IRF8, HR injury could be partially alleviated through inhibition of PINK1/Parkin mitophagy pathway (Fig. 6). However, there are some limitations. First of all, the indexes indicating mitochondrial damage such as membrane potential were not applied. In addition, the signaling pathway upstream of IRF8 was not further investigated. Furthermore, exact death pathways of cells were not further studied. Most importantly, in vivo experiments were also needed to validate the findings of this study. Thus, this is a preliminary study and further research is required for validation.

\section{Conclusions}

The current study reveals that IRF8 could promote cell injury and inflammation during HR, and the effect was exerted through PINK1/Parkin mitophagy pathway. Therefore, IRF8 regulation combined with PINK1 mediated mitophagy inhibition may elucidate a protective mechanism against HR injury of macrophages, with the potential to be a new approach in treating lung ischemia-reperfusion injury.

\section{Abbreviations}

LIRI: lung ischemia-reperfusion injury; PGD: primary graft dysfunction; IRF, interferon regulatory factor; IRF8: interferon regulatory factor 8; mTOR: mammalian target of rapamycin; HR: hypoxia/reoxygenation; PTEN: phosphatase and tensin homologue; PINK1: PTEN)-induced putative kinase 1; NC: normal control; shRNA: short hairpin RNA; sh-IRF8: IRF8 shRNA plasmids; sh-NC: negative control plasmids; OE-PINK1: PINK1 overexpression plasmids; CCK8: cell counting kit-8; LDH: lactate dehydrogenase; ELISA: enzymelinked immunosorbent assay; qPCR: quantitative real-time PCR; PVDF: polyvinylidene fluoride; TEM: transmission electron microscopy; ANOVA: analysis of variance; DBD: DNA binding domain; IAD: IRFassociated domain; OMM: outer membrane of mitochondria; LIR: LC3 interacting region. 


\section{Declarations}

\section{Author contributions}

Conceptualization, Lin Zhang and Bo Hao; Data curation, Bo Hao; Formal analysis, Xiaokang Shen, Ziyao Zhang, Wenyang Jiang and Wei Wang; Funding acquisition, Tao Fan and Qing Geng; Investigation, Zilong Lu and Haojie Feng; Methodology, Lin Zhang, Bo Hao, Ruyuan He, Zilong Lu and Haojie Feng; Project administration, Qing Geng; Resources, Tao Fan; Software, Xiaokang Shen, Ziyao Zhang, Wenyang Jiang and Wei Wang; Visualization, Ruyuan He; Supervision, Qing Geng; Writing - original draft, Lin Zhang; Writing - review \& editing, Tao Fan and Qing Geng.

\section{Acknowledgments}

Not applicable.

\section{Consent for publication}

Not applicable.

\section{Funding}

This project was supported by National Natural Science Foundation of China to Qing Geng (81770095) and to Tao Fan (81700093).

\section{Competing interests}

The authors declared that there is no competing interests.

\section{Availability of data and materials}

The datasets used and/or analysed during the current study are available from the corresponding author on reasonable request.

\section{References}

1. Ferrari RS, Andrade CF: Oxidative Stress and Lung Ischemia-Reperfusion Injury. Oxid Med Cell Longev 2015, 2015:1-14.

2. Wang X, O Brien ME, Yu J, Xu C, Zhang Q, Lu S, Liang L, An X, McDyer JF, Mallampalli RK: Prolonged Cold Ischemia Induces Necroptotic Cell Death in Ischemia-Reperfusion Injury and Contributes to Primary Graft Dysfunction after Lung Transplantation. Am J Resp Cell Mol 2019, 61(2):244-256.

3. Laubach VE, Sharma AK: Mechanisms of lung ischemia-reperfusion injury. Curr Opin Organ Tran 2016, 21(3):246-252.

4. Yanai $H$, Negishi $H$, Taniguchi T: The IRF family of transcription factors: Inception, impact and implications in oncogenesis. Oncoimmunology 2012, 1(8):1376-1386. 
5. Jefferies CA: Regulating IRFs in IFN Driven Disease. Front Immunol 2019, 10.

6. Gupta M, Shin D, Ramakrishna L, Goussetis DJ, Platanias LC, Xiong H, Morse RHC, Ozato K: IRF8 directs stress-induced autophagy in macrophages and promotes clearance of Listeria monocytogenes. Nat Commun 2015, 6(1):6379.

7. Dikic I, Elazar Z: Mechanism and medical implications of mammalian autophagy. Nat Rev Mol Cell Biol 2018, 19(6):349-364.

8. Youle RJ, Narendra DP: Mechanisms of mitophagy. Nat Rev Mol Cell Biol 2011, 12(1):9-14.

9. Liu WC, Chen SB, Liu S, Ling X, Xu QR, Yu BT, Tang J: Inhibition of mitochondrial autophagy protects donor lungs for lung transplantation against ischaemia-reperfusion injury in rats via the mTOR pathway. J Cell Mol Med 2019, 23(5):3190-3201.

10. Zhao M, Fernandez LG, Doctor A, Sharma AK, Zarbock A, Tribble CG, Kron IL, Laubach VE: Alveolar macrophage activation is a key initiation signal for acute lung ischemia-reperfusion injury. $A m J$ Physiol-Lung C 2006, 291(5):L1018-L1026.

11. Fan T, Huang Z, Wang W, Zhang B, Xu Y, Mao Z, Chen L, Hu H, Geng Q: Proteasome inhibition promotes autophagy and protects from endoplasmic reticulum stress in rat alveolar macrophages exposed to hypoxia-reoxygenation injury. J Cell Physio/ 2018, 233(10):6748-6758.

12. Zhang J, Wang J, Zheng Z, Tang J, Fan K, Guo H, Wang J: Participation of autophagy in lung ischemia-reperfusion injury in vivo. J Surg Res 2013, 182(2):e79-e87.

13. Yao L, Chen H, Wu Q, Xie K: Hydrogen-rich saline alleviates inflammation and apoptosis in myocardial I/R injury via PINK-mediated autophagy. Int J Mol Med 2019, 44(3):1048-1062.

14. Zheng J, Chen L, Lu T, Zhang Y, Sui X, Li Y, Huang X, He L, Cai J, Zhou C et al: MSCs ameliorate hepatocellular apoptosis mediated by PINK1-dependent mitophagy in liver ischemia/reperfusion injury through AMPKa activation. Cell Death Dis 2020, 11(4).

15. Palikaras K, Lionaki E, Tavernarakis N: Mechanisms of mitophagy in cellular homeostasis, physiology and pathology. Nat Cell Biol 2018, 20(9):1013-1022.

16. Fan T, Huang Z, Chen L, Wang W, Zhang B, Xu Y, Pan S, Mao Z, Hu H, Geng Q: Associations between autophagy, the ubiquitin-proteasome system and endoplasmic reticulum stress in hypoxiadeoxygenation or ischemia-reperfusion. Eur J Pharmacol 2016, 791:157-167.

17. Fan T, Chen L, Huang Z, Mao Z, Wang W, Zhang B, Xu Y, Pan S, Hu H, Geng Q: Autophagy decreases alveolar macrophage apoptosis by attenuating endoplasmic reticulum stress and oxidative stress. Oncotarget 2016, 7(52):87206-87218.

18. Pak O, Sydykov A, Kosanovic D, Schermuly RT, Dietrich A, Schroder K, Brandes RP, Gudermann T, Sommer N, Weissmann N: Lung Ischaemia-Reperfusion Injury: The Role of Reactive Oxygen Species. Adv Exp Med Biol 2017, 967:195-225.

19. Shologu N, Scully M, Laffey JG, O Toole D: Human Mesenchymal Stem Cell Secretome from Bone Marrow or Adipose-Derived Tissue Sources for Treatment of Hypoxia-Induced Pulmonary Epithelial Injury. Int J Mol Sci 2018, 19(10):2996. 
20. Bernard O, Jeny F, Uzunhan Y, Dondi E, Terfous R, Label R, Sutton A, Larghero J, Vanneaux V, Nunes H et al: Mesenchymal stem cells reduce hypoxia-induced apoptosis in alveolar epithelial cells by modulating HIF and ROS hypoxic signaling. Am J Physiol-Lung C 2018, 314(3):L360-L371.

21. Sharma AK, Fernandez LG, Awad AS, Kron IL, Laubach VE: Proinflammatory response of alveolar epithelial cells is enhanced by alveolar macrophage-produced TNF-a during pulmonary ischemiareperfusion injury. Am J Physiol-Lung C 2007, 293(1):L105-L113.

22. Lee KY, Geng H, Ng KM, Yu J, van Hasselt A, Cao Y, Zeng YX, Wong AH, Wang X, Ying J et al: Epigenetic disruption of interferon-gamma response through silencing the tumor suppressor interferon regulatory factor 8 in nasopharyngeal, esophageal and multiple other carcinomas. Oncogene 2008, 27(39):5267-5276.

23. Guo Y, Lin C, Xu P, Wu S, Fu X, Xia W, Yao M: AGEs Induced Autophagy Impairs Cutaneous Wound Healing via Stimulating Macrophage Polarization to M1 in Diabetes. Sci Rep 2016, 6:36416.

24. Xu H, Zhu J, Smith S, Foldi J, Zhao B, Chung AY, Outtz H, Kitajewski J, Shi C, Weber S et al: NotchRBP-J signaling regulates the transcription factor IRF8 to promote inflammatory macrophage polarization. Nat Immunol 2012, 13(7):642-650.

25. Jia Y, Han S, Li J, Wang H, Liu J, Li N, Yang X, Shi J, Han J, Li Y et al: IRF8 is the target of SIRT1 for the inflammation response in macrophages. Innate Immun-London 2016, 23(2):188-195.

26. Yoshida Y, Yoshimi R, Yoshii H, Kim D, Dey A, Xiong H, Munasinghe J, Yazawa I, O Donovan MJ, Maximova OA et al: The Transcription Factor IRF8 Activates Integrin-Mediated TGF- $\beta$ Signaling and Promotes Neuroinflammation. Immunity 2014, 40(2):187-198.

27. Liu S, Zhang J, Yu B, Huang L, Dai B, Liu J, Tang J: The role of autophagy in lung ischemia/reperfusion injury after lung transplantation in rats. Am J Trans/ Res 2016, 8(8):3593.

28. Ye C, Qi W, Dai S, Zou G, Liu W, Yu B, Tang J: microRNA-223 promotes autophagy to aggravate lung ischemia-reperfusion injury by inhibiting the expression of transcription factor HIF2alpha. Am J Physiol Lung Cell Mol Physiol 2020.

29. Levine B, Ranganathan R: Autophagy: Snapshot of the network. Nature 2010, 466(7302):38-40.

30. Xie WY, Zhou XD, Yang J, Chen LX, Ran DH: Inhibition of autophagy enhances heat-induced apoptosis in human non-small cell lung cancer cells through ER stress pathways. Arch Biochem Biophys 2016, 607:55-66.

31. Villa E, Marchetti S, Ricci J: No Parkin Zone: Mitophagy without Parkin. Trends Cell Bio/ 2018, 28(11):882-895.

\section{Figures}


A
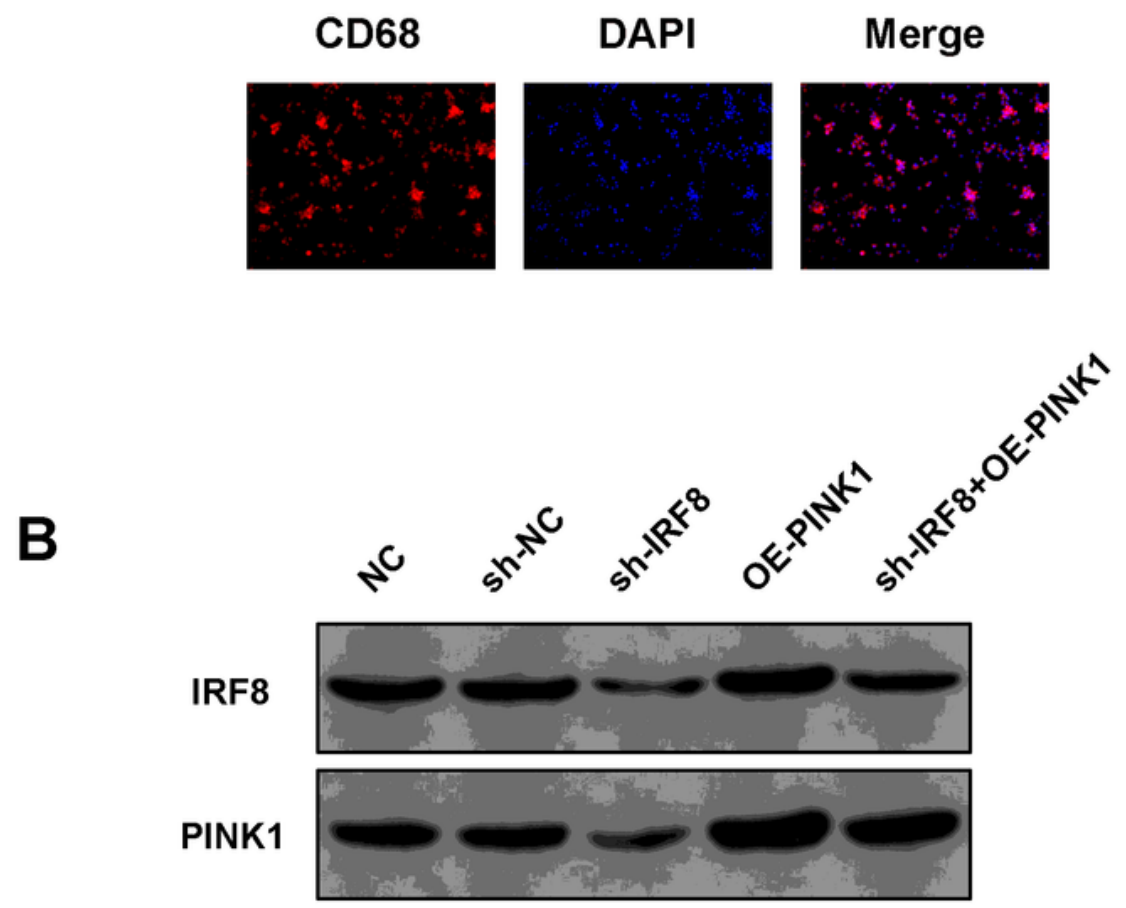

GADPH

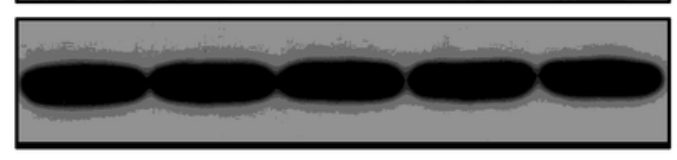

\section{C}
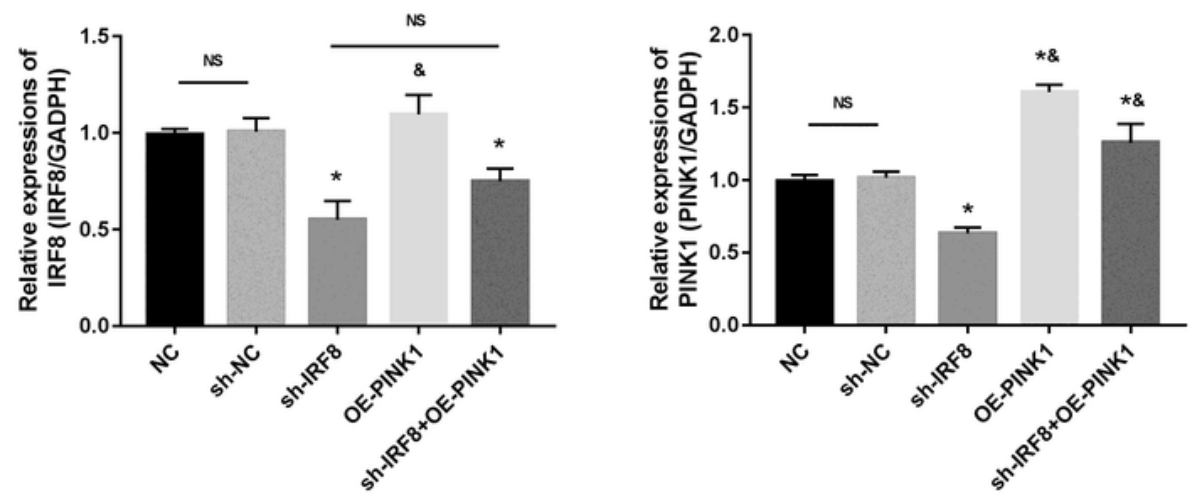

Figure 1

The evidence of IRF8 knock-down and PINK1 overexpression in NR8383 macrophages. A, Immunofluorescence staining of CD68 in cultured cells before transfection and HR treatment. CD68 staining is shown in red, representing macrophages, DAPI staining in the nuclei is shown in blue; the magnification is 200x. B-D, Successful downregulation of IRF8 by sh-IRF8 and upregulation of PINK1 by OE-PINK1 in macrophages. Western blot analyses of IRF8 and PINK1 proteins in NR8383 macrophages 
under normoxia conditions (B) and the densitometric analyses (C,D) of them. Cells were normally cultured as NC or transfected with indicated plasmids before analyses. NS, not significant. NC, normal control. Data are shown as means $\pm S D$. ${ }^{*} P<0.05$, compared with NC group; $\$ P<0.05$, compared with sh-IRF8 group

A

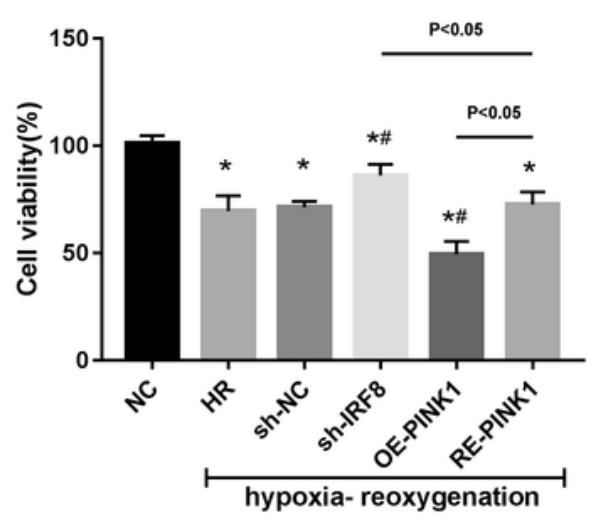

C

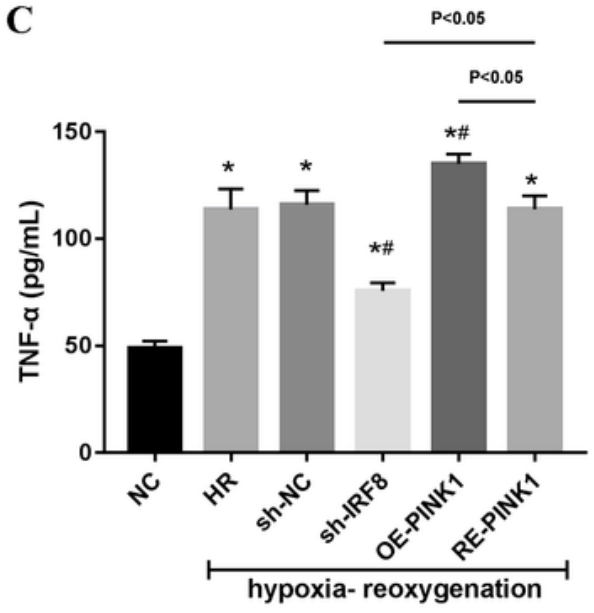

$\mathbf{E}$

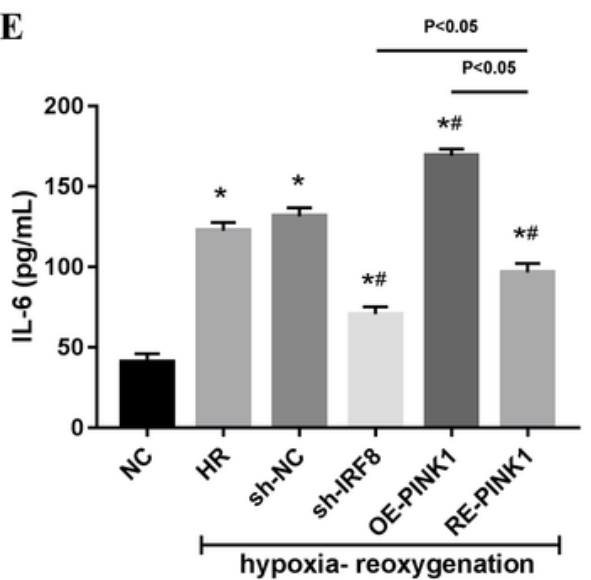

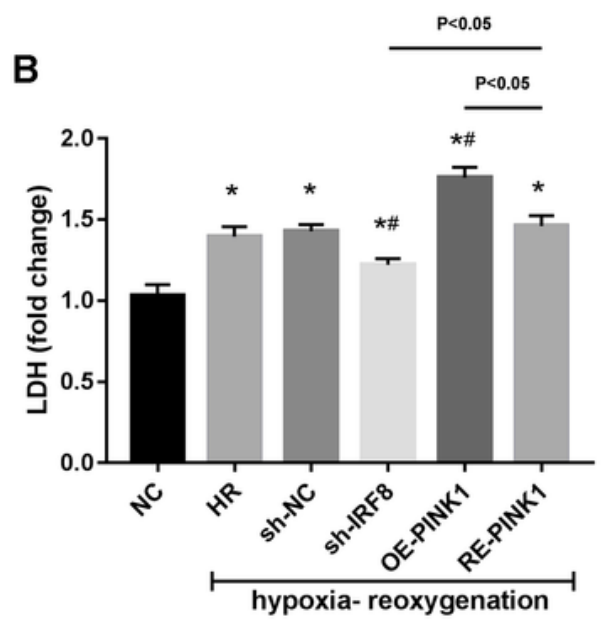

D

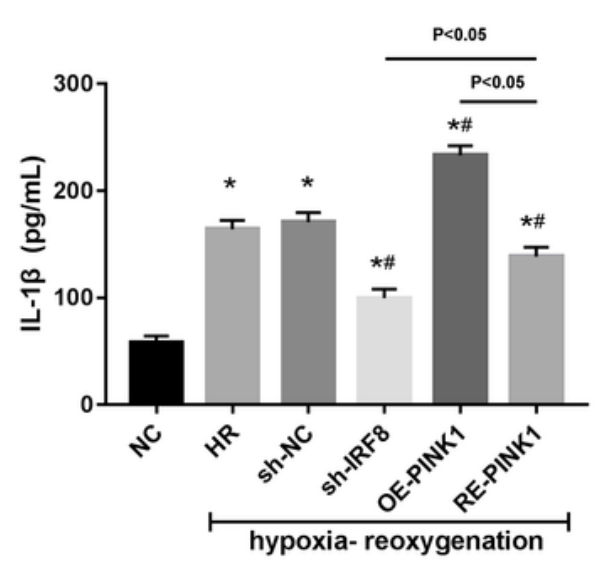

F

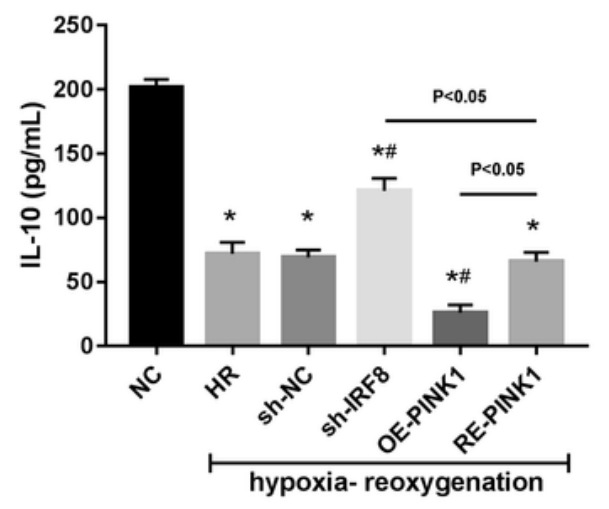

Figure 2 
The effects of IRF8 inhibition on HR-induced cell injury and inflammation in macrophages. A-B, Effects of IRF8 on cell viability, cell damage during HR and the regulatory role of IRF8 in the effects of PINK1 on them. Cell viability was assessed by CCK8 assay (A), cell damage was assessed by LDH activity test (B). $C-F$, Inhibition of IRF8 alleviates inflammation during HR and attenuates the promoting effects of PINK1 on it. TNF- $a(C), I L-1 \beta$ (D), IL-6 (E) and IL-10 (F) were assessed by ELISA in NR8383 macrophages. Cells were subjected to normoxia as $\mathrm{NC}$, underwent HR directly or underwent HR after transfected with indicated plasmids. NC, normal control. HR, hypoxia/reoxygenation. RE-PINK1, sh-IRF8 and OE-PINK1 cotransfection. Data are shown as means $\pm \mathrm{SD}$. ${ }^{*} \mathrm{P}<0.05$, compared with $\mathrm{NC}$ group; $\mathrm{AP}<0.05$, compared with HR group.

A

NC

HR

sh-NC

sh-IRF8

OE-PINK1

RE-PINK1
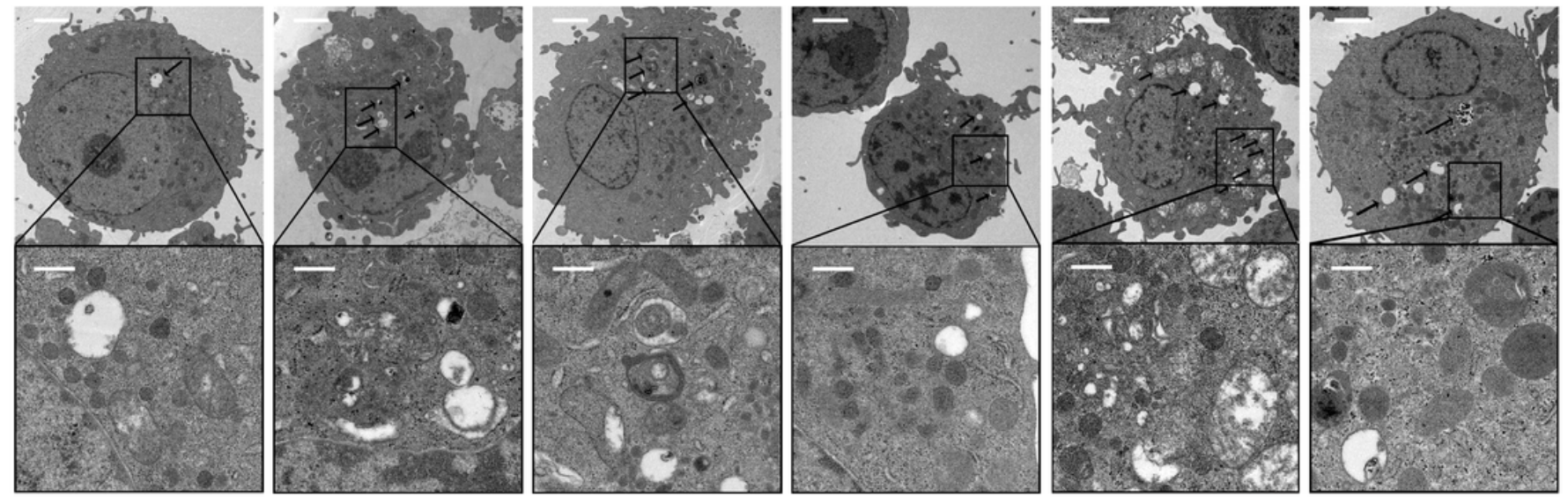

B

C

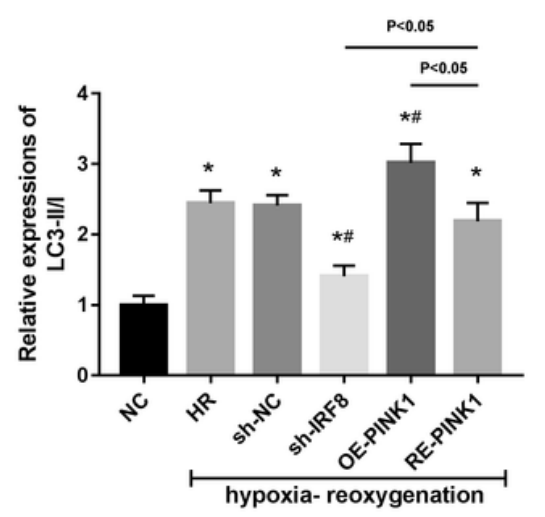

\section{Figure 3}

IRF8 inhibition mitigates autophagy and attenuates the promoting effects of PINK1 on autophagy in macrophages. A, Transmission electron microscope observation of autophagosome formations. Black arrows indicate autophagosomes; the magnification is 1700x and the scale bar is $2 \mu \mathrm{m}$ for upper row images and 7800x, $500 \mathrm{~nm}$ for lower ones. B-C, Expression of LC3 proteins in NR8383 macrophages. Western blot analyses of LC3 (B) and the densitometric analyses of them (C). Cells were subjected to normoxia as NC, underwent HR directly or underwent HR after transfected with indicated plasmids. NC, 
normal control. HR, hypoxia/reoxygenation. RE-PINK1, sh-IRF8 and OE-PINK1 co-transfection. Data are shown as means $\pm \mathrm{SD}$. ${ }^{*} \mathrm{P}<0.05$, compared with $\mathrm{NC}$ group; $\# \mathrm{P}<0.05$, compared with HR group.

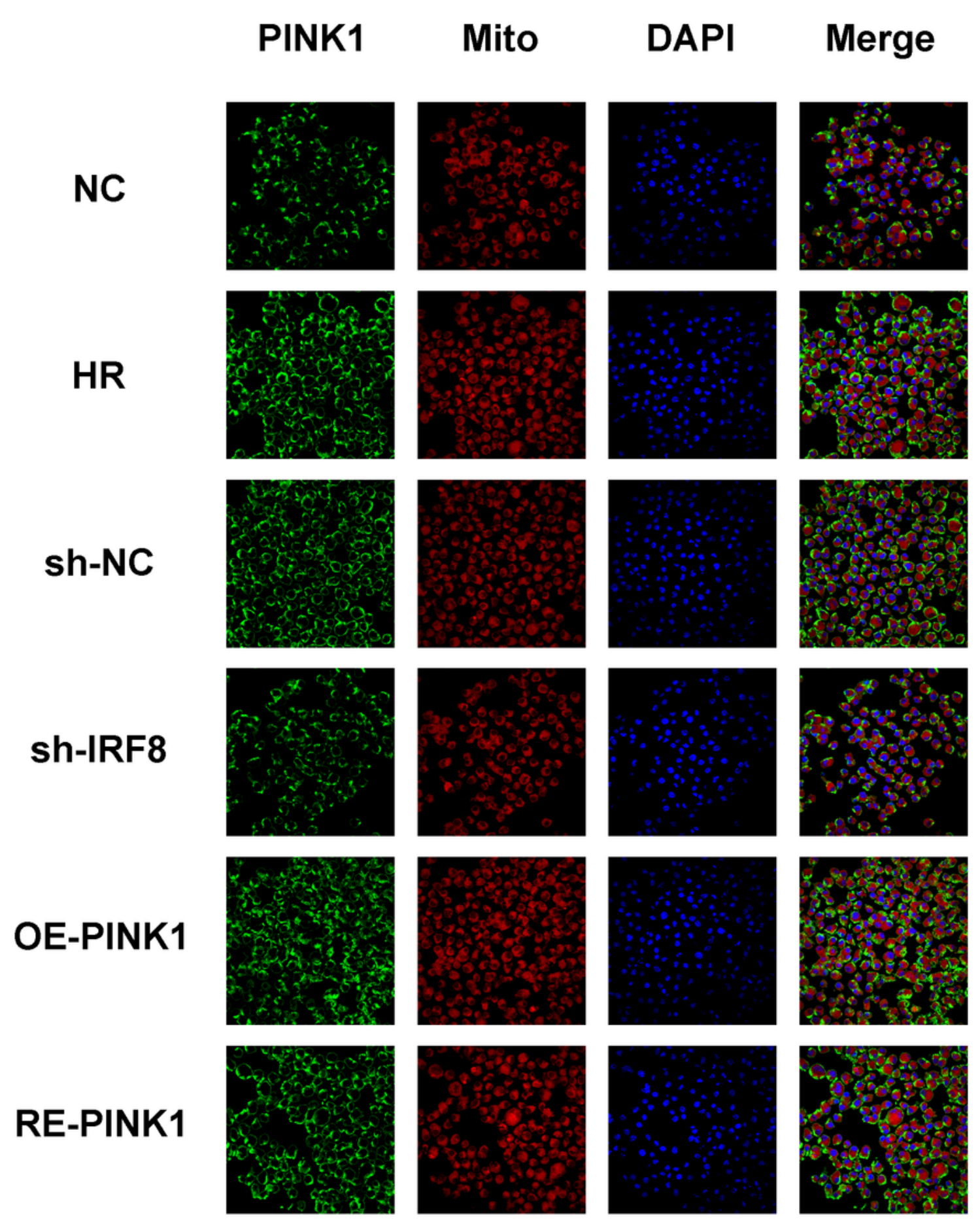

Figure 4

The alleviation of mitophagy by IRF8 knock-down and the regulatory role of IRF8 on the effects of PINK1 on mitophagy in macrophages. Double immunofluorescence labeling images demonstrating the colocalization of PINK1 and mitochondria in NR8383 macrophages. PINK1 staining is shown in green, 
representing the expression of PINK1, DAPI staining in the nuclei is shown in blue, and MitoTracker staining is shown in red, demonstrating the distribution of mitochondria; the magnification is $600 \times$. Cells were subjected to normoxia as NC, underwent HR directly or underwent HR after transfected with indicated plasmids. NC, normal control. HR, hypoxia/reoxygenation. RE-PINK1, sh-IRF8 and OE-PINK1 cotransfection. Mito, MitoTracker, a fluorescent dye demonstrating the distribution of mitochondria.

A

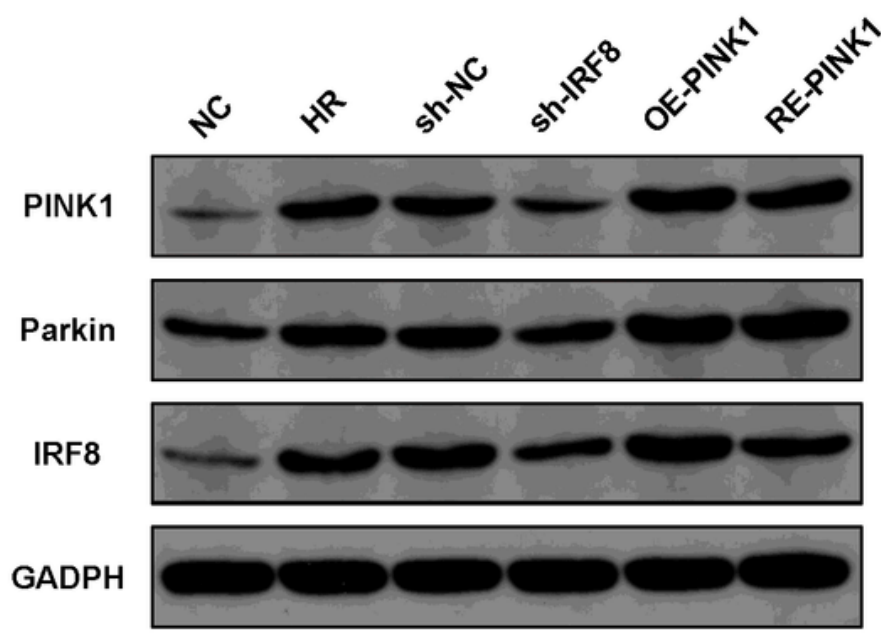

B

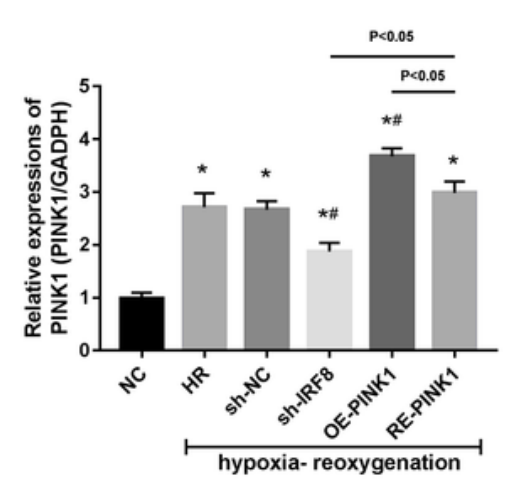

E

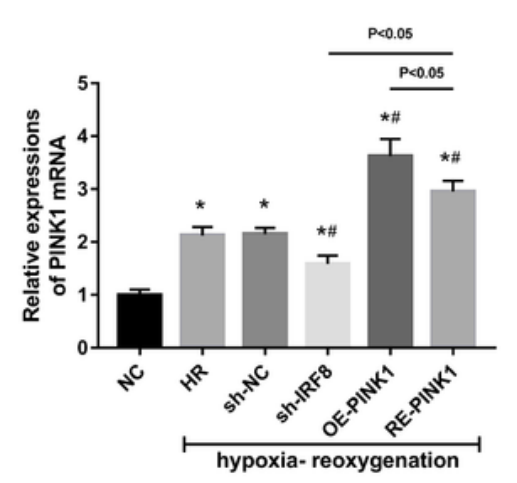

C

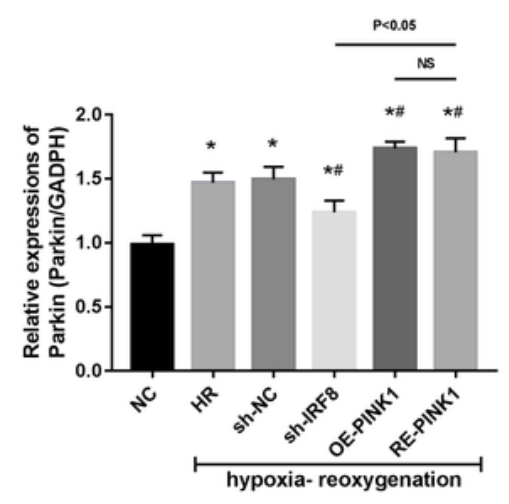

$\mathbf{F}$

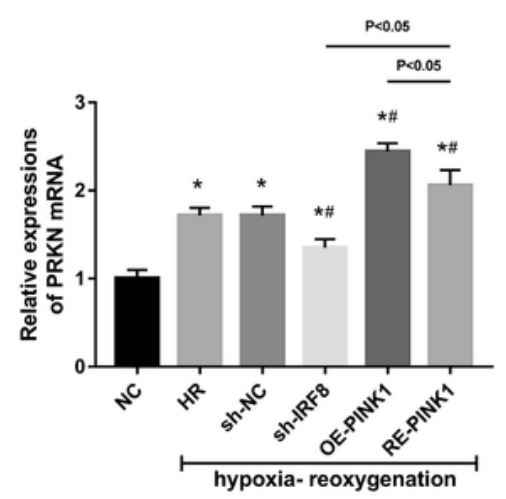

D

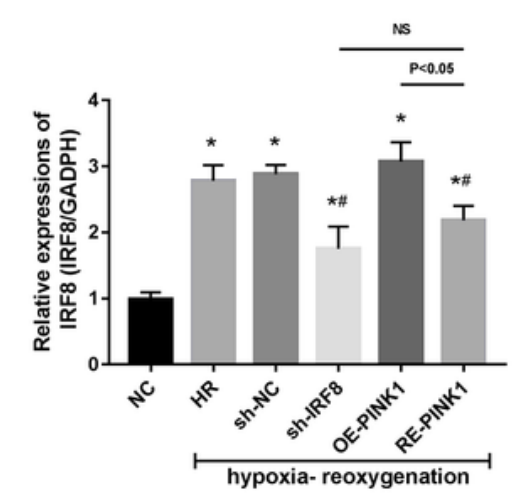

G

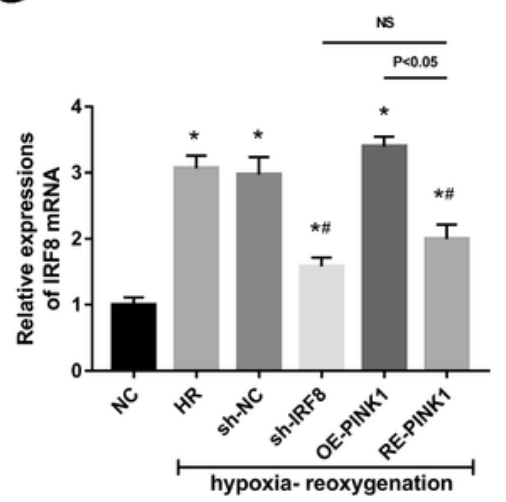

Figure 5 
The effects of IRF8 inhibition on PINK1/Parkin mitophagy pathway. A-G, Effects of IRF8 inhibition on PINK1, Parkin, IRF8 proteins and mRNAs during HR in macrophages and the regulations of IRF8 on the effects of PINK1 on them. Western blot analyses of PINK1, Parkin and IRF8 proteins (A) in NR8383 macrophages and the densitometric analyses of them (B-D). E-G, QPCR analyses of PINK1 (E), Parkin (F) and IRF8 (G) mRNA in macrophages. Cells were subjected to normoxia as NC, subjected to HR directly, or after transfected with indicated plasmids. NS, not significant. NC, normal control. HR, hypoxia/reoxygenation. PRKN, the gene that encodes Parkin proteins. RE-PINK1, sh-IRF8 and OE-PINK1 co-transfection. Data are shown as means $\pm S D$. ${ }^{*} P<0.05$, compared with $N C$ group; $\# P<0.05$, compared with HR group.

\section{Hypoxia/reoxygenation}

\section{IRF8}
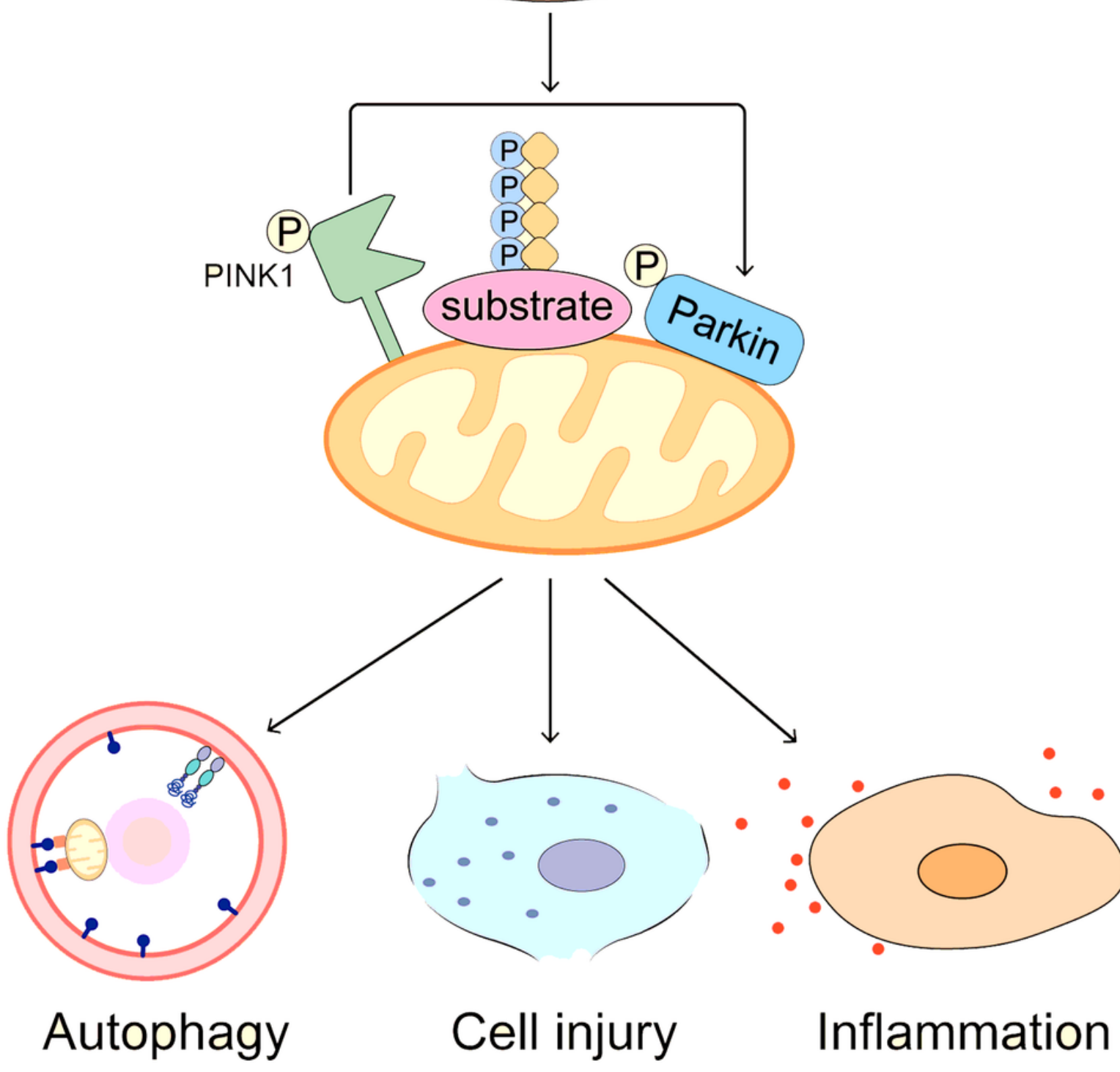

Figure 6 
Schematic illustration of the mechanism underlying the regulatory role of IRF8 in HR-induced, PINK1/Parkin-mediated mitochondrial autophagy, cell injury and inflammation. 